\title{
Résultats d'une intervention de dépistage communautaire de la tuberculose dans un village du Nunavik, au Québec, de 2015 à 2016
}

\author{
R Dion ${ }^{1 *}, \mathrm{M}$ Brisson², JF Proulx², H Zoungrana²
}

\section{Résumé}

Contexte : Une éclosion de tuberculose (TB) s'est produite dans un petit village du Nunavik entre 2012 et 2013 , et a connu une résurgence entre 2015 et 2016 , et ce malgré les suivis des contacts incluant un quart de la population. Une décision d'effectuer un dépistage à grande échelle de la TB a été prise.

Objectif : Décrire les résultats d'une intervention de dépistage communautaire de la TB qui visait à repérer les personnes atteintes d'une infection tuberculeuse latente (ITL) ou de TB active nécessitant une prophylaxie.

Méthodologie : On a recherché les antécédents d'infection tuberculeuse (TB active ou ITL, définie comme un résultat de test cutané à la tuberculine [TCT] d'au moins $5 \mathrm{~mm}$ d'induration, donc positif) et de prophylaxie (jugée adéquate si au moins $80 \%$ des doses prescrites avaient été prises). Le dépistage ciblait les personnes de deux ans et plus qui n'avaient pas été incluses dans les suivis des contacts menées après le 1er juin 2015 ( $n=1026$ personnes admissibles). Le dépistage comprenait une évaluation clinique infirmière, un TCT pour les personnes dont le TCT antérieur était négatif ou de statut inconnu et une radiographie pulmonaire pour les autres personnes.

Résultats : Parmi les personnes admissibles dans le village touché, 1004 (98\%) ont participé au dépistage. De ce chiffre, $30 \%$ avaient des antécédents d'infection tuberculeuse. Un TCT de dépistage a été administré à $71 \%$ des participants, dont $10 \%$ ont obtenu un résultat positif. Des évaluations ont été effectuées chez 425 participants et 385 ont subi une radiographie pulmonaire. Cinquante-deux cas de TB diagnostiqués antérieurement et trois nouveaux cas de TB active ont été répertoriés. De plus, 247 personnes atteintes d'ITL et qui avaient déjà été repérées (191 avaient reçu une prophylaxie adéquate pour I'ITL, 56 avaient reçu une prophylaxie inadéquate) et 69 étaient des cas d'ITL de novo. On a aussi déterminé que 633 participants n'avaient aucune infection tuberculeuse. Une prophylaxie pour ITL a été recommandée pour 125 participants. Des renseignements de suivi étaient disponibles pour 120 d'entre eux; 85 (71\%) ont complété la prophylaxie.

Conclusion : Dans ce village nordique où sévissait une transmission persistante de la TB en dépit de mesures de contrôle classiques, un dépistage de la population a connu un degré élevé de participation et s'est révélé une manière efficace de détecter d'autres cas de TB active et de personnes atteintes d'ITL.
Citation proposée : Dion R, Brisson M, Proulx JF, Zoungrana H. Résultats d'une intervention de dépistage communautaire de la tuberculose dans un village du Nunavik, au Québec, de 2015 à 2016. Relevé des maladies transmissibles au Canada 2018;44(10):292-8. https://doi.org/10.14745/ccdr.v44i10a04f

Mots-clés : tuberculose, Inuit, screening, dépistage, test cutané à la tuberculine, radiographie pulmonaire, infection tuberculeuse latente, prophylaxie antituberculeuse
Affiliations

${ }^{1}$ Institut national de santé publique du Québec, Laboratoire de santé publique du Québec, Sainte-Anne-de-Bellevue (Québec)

2 Department of Public Health, Nunavik Regional Board of Health and Social Services, Kuujjuaq (Québec)

*Correspondance: rejean.dion@ inspq.qc.ca 


\section{Introduction}

Le taux d'incidence de la tuberculose (TB) chez les Inuit est presque 300 fois plus élevé que parmi les non-Autochtones nés au Canada $(1,2)$. Les déterminants de la TB et les défis pour son contrôle dans les communautés Inuit sont, entre autres, la pauvreté, l'insécurité alimentaire, les logements surpeuplés, I'instabilité d'accès à des soins de santé adaptés à la culture, le faible niveau de scolarité, le tabagisme, l'abus d'alcool et de drogues, et la prévalence élevée de plusieurs maladies chroniques $(2,3)$.

Le présent article porte sur une récente éclosion de TB dans un village du Nunavik, au Québec. Le Nunavik représente le tiers de la superficie du Québec et constitue l'une des quatre régions Inuit du Nord du Canada. Ensemble, ces quatre régions forment la région du Nunangat. Les défis associés à la prise en charge de la TB dans une population Inuit éloignée étaient similaires dans ce village. Les caractéristiques socioéconomiques de ce village sont typiques de la plupart des communautés Inuit. La grande majorité $(99,5 \%)$ des habitants de ce village sont d'ascendance autochtone (dans ce cas-ci, Inuit) et la population est relativement jeune (35,5\% étaient âgés de 14 ans ou moins en 2016) (4). Les conditions de logement laissent à désirer : $52 \%$ des logements sont surpeuplés et $24 \%$ ont besoin de réparations majeures (5). Le tabagisme est très répandu au Nunavik : $67 \%$ des habitants disaient fumer régulièrement en 2012 (6). Cette même année, 55 \% des Inuit du Nunangat âgés de 25 ans et plus disaient avoir des problèmes d'insécurité alimentaire. Un pourcentage élevé des dépenses des ménages au Nunavik était consacré à la nourriture (42\%) et pour les foyers à faible revenu, cette proportion était encore plus élevée. L'insécurité alimentaire et les dépenses connexes sont particulièrement courantes dans les communautés plus petites et plus éloignées, étant donné que le seul moyen de transport reliant les villages en toutes saisons est l'avion (7-9).

À cause de ces défis, et malgré les efforts déployés par la santé publique, le taux $d$ 'incidence de TB est à la hausse au Nunavik depuis 2008 (figure 1).

Le village du Nunavik visé par cette étude a connu une éclosion de 31 cas de TB de 2012 à 2013. La TB n'était pas fréquente dans ce village puisqu'aucun cas n'y avait été identifié entre 1990 et 2006. Par après, seulement un cas par année avait été identifié en 2007 et 2008 et aucun en 2009. Toutefois, quelques cas ont été identifiés au cours des deux années précédant cette éclosion (cinq cas en 2010 et huit cas en 2011). À la suite d'une intervention concertée de la santé publique, on estimait que l'éclosion était sous contrôle en 2014. Toutefois, on a observé une résurgence de la maladie en juin 2015 et, au 19 septembre de cette année-là, le nombre cumulatif de cas détectés depuis le début de cette année était de 22. En réponse, la Direction de la santé publique (DSP) du Nunavik et le Centre de santé Inuulitsivik (CSI) ont procédé aux suivis des contacts, qui incluaitent près d'un quart de la population.
Figure 1 : Taux d'incidence quinquennal moyen de la tuberculose au Nunavik, QC, de 1990 à 2017a

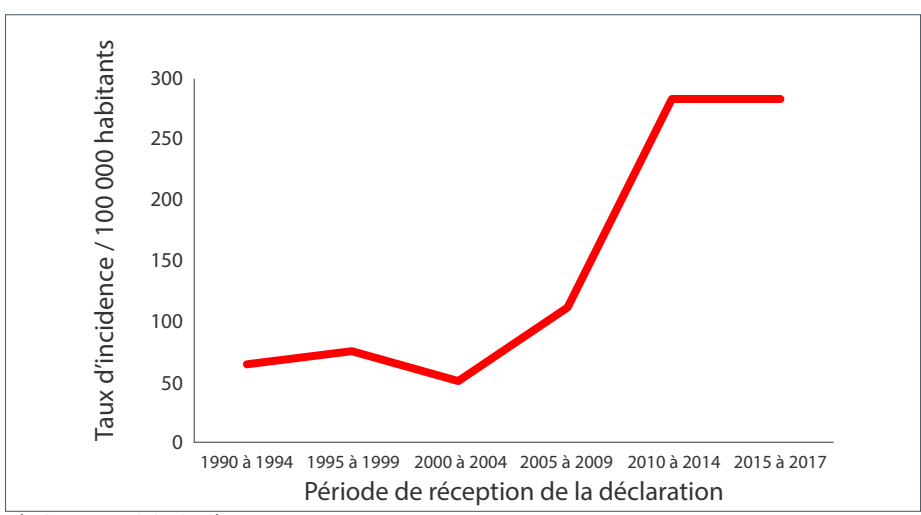

Abréviation : QC, Québec

Sources : Cas de tuberculose : Système des maladies à déclaration obligatoire (MADO), Québec (29 juin 2018). Population : Institut de la statistique du Québec (ISQ). Estimations et projection de la population selon le territoire de région sociosanitaire, 1981 à 2036 (mise à jour : 25 février 2016). Le taux d'incidence moyen de 2015 à 2017 a été calculé sur trois ans

De plus, sur recommandation du Comité sur l'immunisation du Québec (CIQ), la vaccination au bacille Calmette-Guérin (BCG) a été réintroduite chez les enfants de deux ans et moins. (Groupe de travail sur le vaccin BCG. Avis sur la pertinence de la réintroduction du vaccin BCG au Nunavik pour le contrôle d'une recrudescence de la tuberculose. Document de travail. p 1-86. INSPQ; 2015. Document non publié).

Malgré les suivis des contacts extensifs, d'autres cas de TB ont été déclarés par la suite, suggérant une transmission persistante de l'infection.

Considérant le fait que de nouveaux cas avaient été détectés à l'extérieur des suivis des contacts, la DSP a décidé de lancer un vaste programme de dépistage de la TB le 19 octobre 2015. Cette intervention a été réalisée en collaboration avec divers intervenants et partenaires, notamment la DSP, le CSI, la Régie régionale de la santé et des services sociaux (RRSSS) du Nunavik, l'Institut national de santé publique du Québec (INSPQ), le Programme canadien d'épidémiologie de terrain de l'Agence de la santé publique du Canada (ASPC) et la Direction générale de la santé des Premières Nations et des Inuits (DGSPNI) appartenant précédemment à Santé Canada (SC). Les objectifs de l'intervention étaient de sensibiliser et d'informer la communauté, de rehausser les mesures de contrôle et de prévention, de repérer les personnes atteintes de TB active et de leur fournir un traitement antituberculeux sans délai afin d'interrompre la transmission, de détecter les personnes nouvellement atteintes d'une infection tuberculeuse latente (ITL) ou chez qui cette infection n'avait pas été traitée adéquatement et de leur fournir une prophylaxie appropriée.

Le présent article a pour but de décrire ce dépistage communautaire et de déterminer le nombre de cas supplémentaires de TB active et d'ITL détectés, ainsi que le nombre de ces derniers ayant reçu une prophylaxie. 


\section{Méthodologie}

\section{Population}

La population cible comprenait les habitants du village qui étaient âgés d'au moins deux ans après le 15 août 2015 et qui n'avaient pas déjà été inclus dans les suivis des contacts effectuées depuis le $1^{\text {er }}$ juin 2015. La base de données de la population a été constituée par jumelage des fichiers du recensement de la municipalité, du dénombrement des maisonnées de l'Office municipal d'habitation, des dossiers médicaux du CSI et des suivis des contacts de la DSP. Elle a permis d'établir la liste des individus à inclure et de préinscrire les valeurs dans certains champs du questionnaire de dépistage.

\section{Définitions des termes}

Un test cutané à la tuberculine (TCT) avec un résultat positif (au moins $5 \mathrm{~mm}$ ) en l'absence de TB active était classifié comme étant une ITL. Ce seuil a été choisi dans le contexte de l'éclosion continue dans le village. Les ITL étaient aussi classifiées comme étant récentes (intervalle de moins de deux ans entre un résultat négatif et un résultat positif au TCT, avec une différence d'au moins $6 \mathrm{~mm}$ entre les deux), nouvelles (date indéterminée, pas de TCT antérieur ou résultat antérieur négatif au TCT au moins deux ans auparavant) ou anciennes (résultat positif au TCT au moins deux ans auparavant). Les ITL récentes et nouvelles ont été regroupées sous la catégorie de novo. Les ITL étaient réputées avoir reçu une prophylaxie adéquate si cette dernière avait été administrée à $80 \%$ (nombre de doses prises divisé par le nombre de doses prescrites). Les infections tuberculeuses antérieures étaient définies comme étant une TB active si le diagnostic avait été validé comme étant confirmé ou probable d'après les définitions nosologiques des cas faisant l'objet d'une surveillance (10), ou si une ITL avait été documentée par le passé. Une TB active soupçonnée était définie comme la présence d'un ou de plusieurs des symptômes ou signes cliniques suivants : toux inhabituelle durant deux semaines ou plus; fièvre persistante; perte de poids significative (ou absence ou retard d'un gain de poids chez une personne en croissance); ou hémoptysie. Une anomalie clinique non spécifique était définie comme une manifestation clinique non évocatrice de TB active, mais nécessitant un suivi infirmier en vue d'en vérifier la persistance et au besoin une évaluation médicale. Le suivi des contacts était défini comme le traçage des personnes en contact avec des cas de TB afin d'identifier et de traiter tous les cas secondaires de TB et d'ITL pour leur offrir un traitement (3).

\section{Collecte des données}

Un questionnaire uniforme et un guide d'utilisation connexe comprenant les sections suivantes ont été créés pour la collecte des données: renseignements de nature démographique, antécédents d'infection tuberculeuse (dont la TB ou I'ITL), évaluation clinique infirmière, TCT (administration et résultats), radiographie pulmonaire $(\mathrm{R} \times \mathrm{P})$ et évaluation médicale.
Un algorithme clinique pour la procédure de dépistage a été élaboré par la DSP en collaboration avec des partenaires, dont des pneumologues du Réseau universitaire intégré de santé de l'Université McGill à Montréal, au Québec. Le dépistage a ensuite été effectué par des infirmières du Nunavik et il comportait les étapes suivantes :

- vérification et complétion des données cliniques préexistantes, des renseignements de nature démographique (dont l'adresse de voirie);

- vérification des antécédents de TB active (avec l'année la plus récente d'apparition), de TCT et résultat du dernier TCT, de diagnostic d'ITL, d'administration du traitement préventif et sa complétude;

- $\quad$ questions sur la présence de symptômes et de signes cliniques suggestifs de TB active;

- $\quad$ administration du TCT aux personnes sans antécédent d'infection tuberculeuse et ne présentant pas de symptôme ou de signe clinique suggestif de TB active;

- lecture et documentation des résultats du TCT entre 48 et 72 heures après son administration;

- recommandation de RxP pour les personnes avec antécédents de TB active ou d'ITL et pour celles avec un résultat de TCT de dépistage d'au moins $5 \mathrm{~mm}$;

- $\quad$ questions sur l'exposition récente (depuis le $1^{\text {er }}$ janvier 2015) à un cas de TB active hospitalisé;

- $\quad$ suivi en présence d'anomalies cliniques non spécifiques;

- $\quad$ aiguillage immédiat vers des médecins cliniciens des personnes présentant des symptômes ou des signes cliniques suggestifs de TB active pour diagnostic et suivi médical, le cas échéant;

- $\quad$ aiguillage vers des médecins cliniciens des personnes ayant des antécédents d'infection tuberculeuse ou un TCT lors du dépistage d'au moins $5 \mathrm{~mm}$, pour diagnostic et décision quant aux prochaines étapes.

\section{Dépistage et suivi}

Le TCT était administré selon le Protocole d'immunisation du Québec (11); ceux dont la lecture n'avait pas été faite ou qui avait été effectuée plus de 72 heures après son administration étaient repris. Le sujet a soit reçu son congé (évaluation clinique normale et résultat négatif au TCT) ou a été aiguillé vers un médecin clinicien.

Le dépistage a débuté dans l'école secondaire et il s'est poursuivi par quartier et par foyer auprès des personnes amenées au Centre local des services communautaires (CLSC) du village, avec l'aide des services de transport municipaux.

\section{Analyse}

Les données ont été saisies dans un fichier Microsoft Access 2007 (Redmond, État de Washington, É-U) et validées par la DSP. Une révision des dossiers médicaux du CLSC a été effectuée à la fin de l'intervention. Enfin, les enregistrements des personnes identifiées comme candidates à la prophylaxie ont été jumelés à une base de données de suivis de traitement d'ITL. Les données 
ont été analysées au moyen des logiciels Epi Info 7.1.5.2 (Centers for Disease Control and Prevention, Atlanta, Georgia, É-U) (12) et SPSS 23 (IBM Analytics, Armonk, New York, É-U) (13).

\section{Résultats}

La population totale du village était estimée à 1477 personnes (Agence de la santé publique du Canada. Shane A, Born J.

Dépistage de la tuberculose en milieu scolaire et dans la population au Nunavik [Québec], du 6 octobre au 13 novembre 2015. p 1-38. ASPC; 2015. Rapport non publié). Deux groupes étaient exclus du dépistage de la TB : 69 enfants âgés de moins de deux ans qui avaient reçu le vaccin BCG et 350 personnes qui avaient déjà été repérées dans le cadre des suivis des contacts. Trente-deux autres personnes ont été éliminées pour diverses raisons (dont 18 personnes qui vivaient à l'extérieur du village et 14 qui étaient hospitalisées ou incarcérées). Le nombre de personnes admissibles était donc de 1026 . De ce chiffre, deux ont refusé de participer et 20 n'ont pu être jointes.

Des évaluations cliniques ont été effectuées sur les 1004 personnes restantes entre le 19 octobre 2015 et le 21 mars 2016 (figure 2).

Figure 2 : Nombre de cas de tuberculose confirmés et probables dans un village selon la date de notification, Nunavik, QC, de 2010 à $2017^{a}$
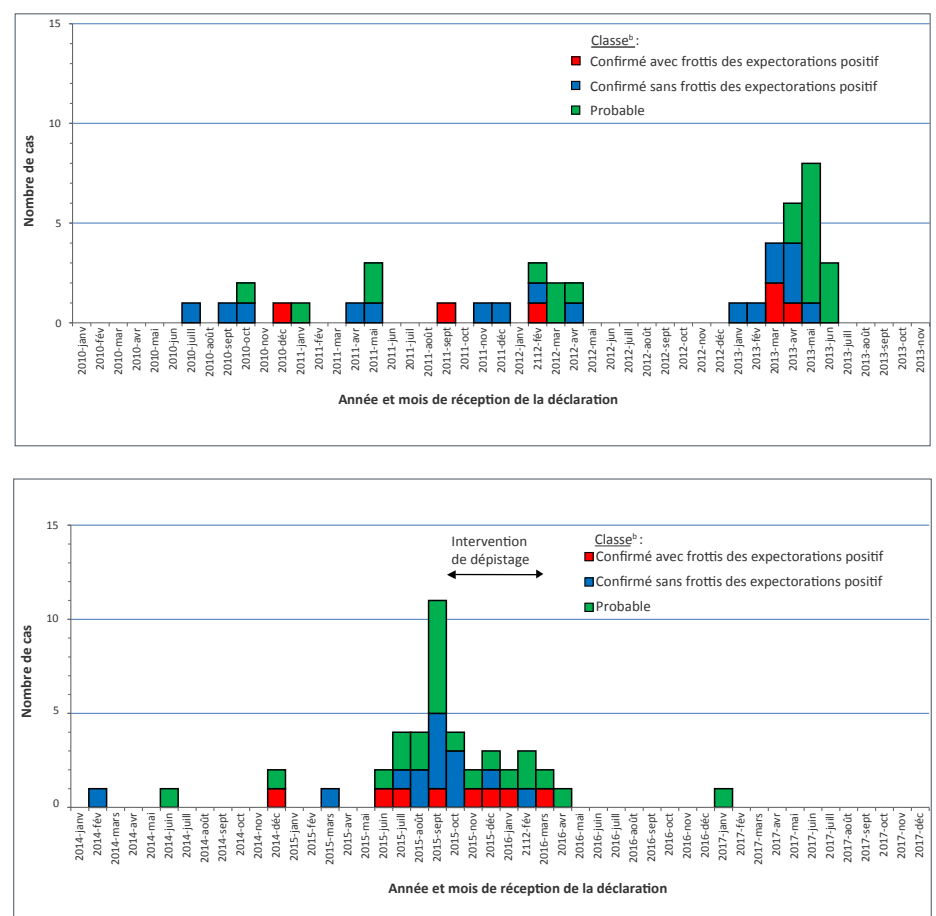

Abréviation : QC, Québec

Source : Système des MADO, Québec (29 juin 2018)

Selon les définitions nosologiques québécoises en vigueur (10)
Quatre-vingt-dix personnes (73,8 \% de la population de 122 élèves, ou 9,0\% du groupe d'étude total) ont fait l'objet d'un dépistage à l'école et 914 (91,0 \%) ont subi leur test de dépistage par maisonnées.

Parmi les 1004 participants, 531 (52,9\%) étaient de sexe masculin et $473(47,1 \%)$ étaient de sexe féminin. Leur âge variait de 20 mois à 85 ans (moyenne : 27,0 ans; médiane : 23,0 ans), avec une distribution semblable chez les deux sexes. Six des participants étaient des enfants de moins de deux ans dont les parents avaient refusé le vaccin BCG antérieurement.

Cinquante-deux (7,8 \%) des 667 personnes pour lesquelles des renseignements étaient disponibles avaient des antécédents de TB active. L'année du diagnostic de TB était disponible pour 44 des cas et elle s'échelonnait de 1955 à 2015. Six cent-sept personnes avaient déjà un résultat positif au TCT dans leur dossier; 282 (46,5 \%) d'entre elles ont obtenu un résultat positif au TCT et, de ce chiffre, 235 (83,3\%) avaient un résultat d'au moins $10 \mathrm{~mm}$ (tableau 1).

Tableau 1 : Distribution des résultats antérieurs aux tests cutanés à la tuberculine au Nunavik, QC

\begin{tabular}{|l|r|r|}
\hline \multicolumn{1}{|c|}{$\begin{array}{c}\text { Résultat de TCT } \\
(\mathrm{mm})\end{array}$} & Nombre & Pourcentage (\%) \\
\hline 0 & 281 & 46,3 \\
\hline 1 à 4 & 44 & 7,2 \\
\hline 5 à 9 & 47 & 7,7 \\
\hline$\geq 10$ & 235 & 38,7 \\
\hline Total & 607 & $100,0^{\circ}$ \\
\hline
\end{tabular}

Abréviations : mm, millimètre; $\mathrm{QC}$, Québec; $\mathrm{TCT}$, test cutané à la tuberculine; $\geq$, supérieur ou égal à

Nombre totalise pas $100 \%$ à cause des valeurs arrondies

La proportion de résultats positifs était semblable selon le sexe, mais elle variait selon l'âge, avec un maximum chez les personnes de 50 ans et plus, suivi des jeunes adultes et des adolescents (figure 3 )

Figure 3 : Résultats antérieurs au test cutané à la tuberculine : Nombre de $5 \mathrm{~mm}$ et plus ou de moins de $5 \mathrm{~mm}$, et proportion de ceux d'au moins $5 \mathrm{~mm}$ selon le groupe d'âge, Nunavik, QC ( $n=607)$

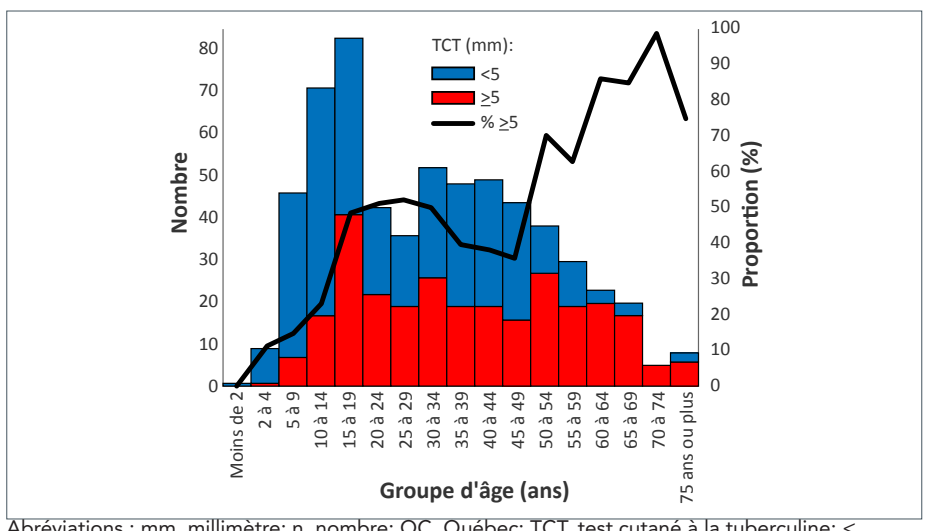

: mm, millimètre; $\mathrm{n}$,

RMTC • Le 4 octobre $42018 \bullet$ Volume 44-10

Page 295 
La prophylaxie était jugée adéquate chez 191 (77,3 \%) des 247 personnes ayant eu un TCT antérieur positif et pour lesquelles I'information était disponible. En incluant les TB actives et les TCT positifs (valeurs non mutuellement exclusives), 297 (29,6\%) des 1004 participants avaient des antécédents d'infection tuberculeuse.

En outre, 47 (4,7\%) des participants ont rapporté un ou plusieurs symptômes ou signes cliniques suggestifs d'une TB active, mais celle-ci n'avait été diagnostiquée chez aucun d'entre eux.

Un TCT de dépistage a été réalisé chez 713 (71,0\%) des 1004 participants, dont 10 ont été refaits par erreur chez des personnes ayant obtenu un résultat antérieur positif au TCT; $85(11,9 \%)$ personnes avaient un résultat positif. Parmi ces dernières, 60 (70,6\%) avaient un résultat d'au moins $10 \mathrm{~mm}$ (tableau 2).

Tableau 2 : Distribution des résultats de TCT de dépistage, Nunavik, QC de 2015 à 2016

\begin{tabular}{|c|c|c|}
\hline $\begin{array}{l}\text { Résultat du TCT } \\
(\mathrm{mm})\end{array}$ & Nombre & Pourcentage (\%) \\
\hline 0 & 562 & 78,8 \\
\hline 1 à 4 & 66 & 9,3 \\
\hline 5 à 9 & 25 & 3,5 \\
\hline$\geq 10$ & 60 & 8,4 \\
\hline Total & 713 & 100 \\
\hline
\end{tabular}

Abréviations : mm, millimètre ; $\mathrm{QC}$, Québec; TCT, test cutané à la tuberculine; $\geq$, supérieur ou égal à

Bien que la proportion de résultats positifs était semblable chez les sujets de sexes masculin et féminins, elle variait selon l'âge, atteignant son maximum chez les personnes de 55 à 64 ans (figure 4).

Figure 4 : Proportion des résultats de TCT de dépistage de $5 \mathrm{~mm}$ et plus et nombre de ceux de moins de $5 \mathrm{~mm}$ selon le groupe d'âge, Nunavik, QC, de 2015 à 2016 $(n=713)$

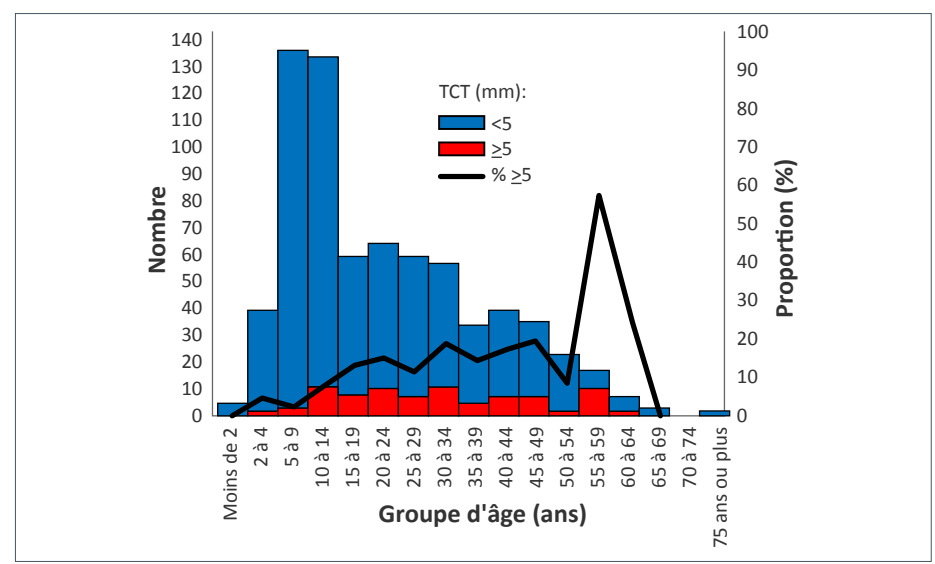

Abréviations : mm, millimètre; $\mathrm{n}$, nombre; $\mathrm{QC}$, Québec; $\mathrm{TCT}$, test cutané à la tuberculine; $<$, inférieur à; $\geq$, supérieur ou égal à
L'intervalle entre l'administration du TCT et sa lecture était de deux jours chez 650 (91,3\%) et de trois jours chez $62(8,7 \%)$ des 712 personnes pour lesquelles les dates étaient connues, donc selon l'intervalle recommandé pour tous ces sujets.

Une RxP a été réalisée chez 385 (38,3\%) des participants. Les résultats étaient classés comme anormaux pour 103 personnes $(26,7 \%)$. Les résultats de la RxP étaient disponibles pour 96 individus, incluant un cas avec un diagnostic de TB pulmonaire active et présentant une lésion cavitaire. Enfin, une évaluation médicale a été réalisée pour 394 (39,2 \%) des participants.

Après vérification des renseignements consignés dans les dossiers cliniques au 12 novembre 2016, les résultats étaient les suivants (tableau 3) : 55 TB actives, soit 52 antérieures et trois nouvelles (deux confirmées et une probable) détectées par le dépistage. En outre, il y avait 316 ITL, incluant 247 anciennes, dont 191 (77,3 \%) avaient reçu une prophylaxie adéquate et 56 $(22,7 \%)$ avaient reçu une prophylaxie incomplète, et 69 avec une ITL de novo; 633 participants ne présentaient aucune infection tuberculeuse.

Tableau 3 : Sommaire des résultats de dépistage de la TB, Nunavik, QC, de 2015 à 2016

\begin{tabular}{|l|r|}
\hline \multicolumn{1}{|c|}{ État } & \multicolumn{2}{|c|}{ Nombre de sujets } \\
\hline TB active & 55 \\
\hline - diagnostiquée antérieurement & 52 \\
\hline - nouvelle détectée par dépistage & 3 \\
\hline ITL & 316 \\
\hline - de novo & $69^{a}$ \\
\hline - diagnostiquée antérieurement & 247 \\
\hline - traité adéquatement & 191 \\
\hline - traitment incomplet & $56^{\text {a }}$ \\
\hline Aucune infection tuberculeuse & 633 \\
\hline Total & 1004 \\
\hline
\end{tabular}

Abréviations : ITL, infection tuberculeuse latente; $\mathrm{OC}$, Québec; TB, tuberculose

a Ces 125 personnes étaient considérées comme candidates à une prophylaxie antituberculeuse: celle-ci a été offerte à 68 des 69 personnes avec une ITL de novo. Des informations sur le suivi de traitement préventif étaient disponibles pour $120(96,0 \%)$ de ces 125 candidats (voir le tableau 4)

Parmi les 125 participants à qui on a offert une prophylaxie antituberculeuse (dont 62 de novo et 56 qui avaient précédemment reçu un traitement incomplet), des dossiers ont été trouvés pour 120 (96,0\%) d'entre eux (tableau 4). De ce nombre, 85 (70,8 \%) ont reçu une prophylaxie antituberculeuse, notamment la rifampicine (94\%) en prise quotidienne durant quatre mois.

La courbe épidémique (figure 2) indique que le dernier cas de l'éclosion de TB de 2015 à 2016 est survenu en avril 2016, pour un cumul de 39 cas, d'aucun n'est décédé. Un cas sporadique a été déclaré en janvier 2017 et deux cas ont été déclarés en mai 2018 (non illustrés ici). 
Tableau 4 : Sommaire des résultats de la prophylaxie antituberculeuse dans un village au Nunavik, $\mathrm{QC}$, de 2015 à 2016

\begin{tabular}{|l|r|r|}
\hline \multicolumn{1}{|c|}{ Issues de la prophylaxie pour ITL } & Nombre & $\begin{array}{c}\text { Pourcentage } \\
\text { (\%) }\end{array}$ \\
\hline $\begin{array}{l}\text { Complétée (au moins } 80 \% \text { des doses } \\
\text { prescrites ont été prises) }\end{array}$ & 85 & 70,8 \\
\hline Reçue mais degré de complétude inconnu & 2 & 1,7 \\
\hline $\begin{array}{l}\text { Débutée mais sujet perdu au suivi } \\
\text { (déménagé du village) }\end{array}$ & 1 & 0,8 \\
\hline Refusée ou cessée par le patient & 13 & 10,8 \\
\hline Cessée par le personnel infirmier & 16 & 13,3 \\
\hline Cessée pour une raison inconnue & 3 & 2,5 \\
\hline Total & 120 & $100,0^{\text {a }}$ \\
\hline
\end{tabular}

Abréviations : ITL, infection tuberculeuse latente; QC, Québec

${ }^{a} \mathrm{Ne}$ totalise pas $100 \%$ à cause des valeurs arrondi

Source : Base de données de suivi de la prophylaxie antituberculeuse, Direction de la santé publique du Nunavik (26 juin 2018)

\section{Discussion}

Presque toute la population de ce village inuit du Nunavik a fait l'objet d'une évaluation pour la TB, que ce soit par suivi des contacts ou dans le cadre de l'intervention de dépistage. Cette intervention d'envergure dans le cadre de laquelle des tests de dépistage ont été administrés à plus de 1000 personnes a été lancée parce qu'on avait observé que l'infection continuait de se transmettre, et ce, malgré l'application de l'approche classique de lutte contre la TB, telle que décrite dans les Normes canadiennes pour la lutte antituberculeuse (3) et le Guide d'intervention contre la tuberculose du Québec (14). Grâce à cette intervention, trois ( $8 \%$ ) des 39 nouveaux cas de TB active qui ont été repérés pendant l'éclosion ont été détectés à travers le dépistage et sans ce dernier, ces cas n'auraient pas été repérés ou ne l'auraient été que plus tard.

Un des points forts de cette intervention est le fait que $98 \%$ des sujets admissibles ont fait l'objet d'un dépistage, ce qui constitue une réalisation remarquable. Le taux élevé de participation obtenu a été possible grâce à la mobilisation du village, à la participation des intervenants et au soutien des divers partenaires et des parties prenantes.

Cette étude avait deux principales limites. La première avait trait aux incertitudes entourant les données démographiques utilisées pour déterminer le sous-groupe devant faire l'objet du dépistage. Quand la base de données sur la population a été créée, aucune des listes disponibles n'était complète. Toutefois, en comparant des listes multiples, il a été possible d'obtenir des approximations raisonnables pour cette population. La seconde limite avait trait au manque d'uniformité et d'intégralité dans la collecte et la saisie des données. Les questionnaires de dépistage n'étaient pas toujours complets puisqu'une partie des renseignements avaient été consignés ailleurs, p. ex. dans les dossiers médicaux. Beaucoup d'intervenants ont participé à I'intervention, ce qui a rendu l'échange d'information difficile, et certaines questions et variables étaient ambiguës ou mal définies. Heureusement, l'examen des renseignements contenus dans les dossiers médicaux a permis d'améliorer l'exactitude des données.

Quoique certains éléments du processus aient été évalués, ces résultats ne mesuraient pas l'impact de l'intervention en termes de cas de TB évités, puisque plusieurs autres facteurs entrent en jeu. Toutefois, le repérage d'un bassin de personnes atteintes d'une ITL de novo ou d'une ancienne ITL avec un traitement incomplet a permis de leur offrir une prophylaxie afin d'éviter qu'elles contractent la maladie et qu'elles deviennent contagieuses à leur tour.

Pour ce qui est des prochaines étapes, un système régional intégré de surveillance de la TB est en voie d'élaboration, ce qui augmentera la capacité de documenter les éclosions de TB et de soutenir les suivis des contacts et le suivi de la prophylaxie et du traitement antituberculeux (15).

\section{Conclusion}

Le dépistage communautaire visant à freiner une éclosion continue de TB dans un village éloigné du Nunavik a permis de joindre $98 \%$ de la population admissible. Il a permis de repérer les sujets qui avaient besoin de traitement et la majorité de ces derniers ont suivi un traitement complet. Les avantages découlant d'une intervention de cette envergure ont été rendus possibles grâce à la capacité du village et des intervenants professionnels, municipaux et du village de fournir un soutien efficace à cette action.

Cet effort intensif a permis de cerner de nouvelles exigences pour la collecte et le stockage de données, ainsi que l'accès à ces dernières, et plusieurs de ces exigences seront résolues par le nouveau système régional intégré de surveillance de la TB. Un tel système facilitera l'évaluation de l'impact des programmes de prévention et de contrôle de la TB dans cette région à l'avenir.

\section{Déclaration des auteurs}

R. D. - Conceptualisation, méthodologie, logiciel, validation, analyse officielle, conservation des données, rédaction - ébauche initiale, étalage

M. B. - Conceptualisation, méthodologie, logiciel, validation, analyse officielle, enquêtes, ressources, conservation des données, rédaction - ébauche initiale, supervision, administration du projet

J. F. P. - Conceptualisation, méthodologie, ressources, rédaction - examen et révision, supervision, administration du projet H. Z. - Conceptualisation, méthodologie, validation, enquêtes, rédaction - examen et révision 


\section{Conflit d'intérêts}

Aucun.

\section{Remerciements}

Nous aimerions remercier les organismes suivants pour leurs contributions au succès de cette intervention : DSP du Nunavik; RRSSS du Nunavik; Réseau universitaire intégré de santé de I'Université McGill; CSI; CLSC du village; DGSPNI de SC; la Direction des risques biologiques et de la santé au travail et le Laboratoire de santé publique du Québec de I'INSPQ; le Programme canadien d'épidémiologie de terrain de I'ASPC; les bénévoles, les interprètes et les responsables de la mobilisation du village, ainsi que le conseil municipal de ce dernier. Nous aimerions également remercier la docteure Marie Rochette, coordonnatrice de la protection de la santé publique à la DSP du Nunavik, pour ses commentaires sur l'ébauche d'article.

\section{Acquisition de financement}

Ce travail a été réalisé grâce au soutien de l'Institut national de santé publique du Québec et de la Direction de la santé publique du Nunavik.

\section{Références}

1. Vachon J, Gallant V, Siu W. La tuberculose au Canada, 2016. Relevé des maladies transmissible au Canada 2018;44(3/4):85-91. https://www.canada.ca/fr/santepublique/services/rapports-publications/releve-maladiestransmissibles-canada-rmtc/numero-mensuel/2018-44/ numero-3-4-1-mars-2018/article-1-tuberculose-2016.html

2. Patterson M, Flinn S, Barker K. Lutter contre la tuberculose chez les Inuit au Canada. Relevé des maladies transmissibles au Canada 2018;44(3/4):92-5. https://www.canada. $\mathrm{ca} / \mathrm{fr} /$ sante-publique/services/rapports-publications/ releve-maladies-transmissibles-canada-rmtc/numeromensuel/2018-44/numero-3-4-1-mars-2018/article-3tuberculose-chez-inuit.html

3. Agence de la santé publique du Canada. Normes Canadiennes pour la lutte antituberculeuse 7e édition: 2014. https://www.canada.ca/fr/sante-publique/services/maladiesinfectieuses/normes-canadiennes-lutte-antituberculeuse-7eedition.html

4. Statistiques Canada. Profil du recensement, Recensement de 2016 (Catalogue no. 98-316-X2016001). Statistiques Canada; 2017. https://www12.statcan.gc.ca/censusrecensement/2016/dp-pd/prof/index.cfm?Lang=F
5. Statistics Canada. Census of Population, 2016. The housing conditions of Aboriginal people in Canada. 1-8. StatsCan; 2017. http://www12.statcan.gc.ca/ census-recensement/2016/as-sa/98-200-x/2016021/98-200x2016021-eng.pdf

6. Bougie E, Kohen D. Smoking prevalence among Inuit in Canada. Health Reports. February. Statistics Canada. 2017. https://www150.statcan.gc.ca/n1/en/pub/82-003-x/2017002/ article/14773-eng.pdf?st=Sr1ML834

7. Arriagada P. Food insecurity among Inuit living in Inuit Nunangat. Insights on Canadian Society. February. Statistics Canada. 2017. https://www150.statcan.gc.ca/n1/pub/75006-x/2017001/article/14774-eng.pdf

8. Blanchet C, Rochette L. Nutrition and Food Consumption among the Inuit of Nunavik. Nunavik Inuit Health Survey 2004, Qanuippitaa? How are we? Institut national de santé publique du Québec (INSPQ) \& Nunavik Regional Board of Health and Social Services (NRBHSS). 2008. https://www. inspq.qc.ca/sites/default/files/publications/762_esi_nutrition_ summary.pdf

9. Robitaille J, Guénard E, Lévesque S, Duhaime G. The cost of living in Nunavik in 2016, Research Report - Revised and Expanded Version. Quebec, Canada Research Chair on Comparative Aboriginal Conditions, Université Laval: 2018. p 1-77. www.nunivaat.org/documents/Publication/ the-cost-of-living-in-nunavik-in-2016-revised-and-expanded. pdf

10. Ministère de la Santé et des Services sociaux. Surveillance des maladies à déclaration obligatoire au Québec. Définitions nosologiques, $11^{\mathrm{e}}$ édition. Maladies d'origine infectieuses. May 2018. http://publications.msss.gouv.qc.ca/ msss/fichiers/2018/18-268-02W.pdf

11. Ministère de la Santé et des Services sociaux. TCT: test cutané à la tuberculine. Protocole d'immunisation du Québec (PIQ). $7^{\text {th }}$ édition. 2018. www.msss. gouv.qc.ca/professionnels/vaccination/piq-vaccins/ vaccins-et-test-cutane-a-la-tuberculine/

12. Centers for Disease Control and Prevention (CDC). Epi Info ${ }^{\text {TM }} .7$ for Windows. www.cdc.gov/epiinfo/index.html

13. International Business Machines (IBM) Corporation. Statistical Package for the Social Science (SPSS). www.ibm.com/ca-en/ marketplace/spss-statistics

14. Ministère de la Santé et des Services sociaux. Guide d'intervention. La tuberculose. 2017. August 2017. http:// publications.msss.gouv.qc.ca/msss/fichiers/2017/17-27105W.pdf

15. Institut national de santé publique du Québec. Outil pour la gestion des éclosions de tuberculose au Nunavik (OGÉTN). https://www.inspq.qc.ca/outil-pour-la-gestion-des-ecl osions-de-tuberculose-au-nunavikRatia iliquis aut dit pro dit aut delibus, con cus es aperi rene inulpa cullabor sus, excea dolores sinum de praerum qui dolorest, voluptae velique pre, voleste mpercie ndignatur, to bea sint. 